\title{
LIFSHITZ-LIKE ARGUMENT FOR LOW-LYING STATES IN A STRONG MAGNETIC FIELD
}

\author{
Cyril FURTLEHNER \\ Institute of Physics, University of Oslo \\ P.O. Box 1048 Blindern \\ N-0316 Oslo, Norway
}

\begin{abstract}
:
We are interested in the question of the localization of an electron moving in two dimensions, submitted to a strong magnetic field and scattered by randomly distributed zero-range impurities. Considering the explicit expression for the density of states obtained by Brézin, Gross and Itzykson, we adapt the Lifshitz argument, in order to analyse the somewhat unusual power-law behavior of the low energy spectrum. The typical configurations of disorder which gives rise to low energy states are identified as cluster of impurities of well defined form, when the impurity density is smaller than the Landau degeneracy. This allows for an interpretation of low lying states, localized around these clusters. The size of these clusters diverges logarithmically when the energy goes to zero.
\end{abstract}




\section{Introduction}

The two dimensionnal problem of an electron submitted to a strong magnetic field and moving in a random potential, has been the subject of intensive investigations, because of its relevance for the integer quantum Hall effect. In the case of a locally correlated disordered potential, some explicit results have been found, concerning the average density of states [1, 2, 3]. Although the DOS doesn't contain in general any information about localization, exception should be made for the tails of the spectrum, which are generally associated with unprobable realizations of the random potential, and as in the Lifshitz-tail examples, are interpreted in terms of localized states. In the strong magnetic field problem, such a situation is encountered in the case of gaussian fluctuations, where the spectrum displays a gaussian tail at large energy [1]. If disorder is realized by delta impurities obeying Poisson statistics, the situation is very different. The spectrum is bounded from below, and instead of having a tail, it is singular at low energy. More precisely, depending on a parameter $f=\frac{\rho}{\rho_{l}}$ which is the ratio between the density of impurities and the Landau degeneracy, it takes the following asymptotic form [2],

$$
\lambda \rho(E) \sim_{\omega \rightarrow+0} \begin{cases}(1-f) \delta(\omega)+A(f) \omega^{-f}, & 0<f<1 \\ \frac{1}{\omega(\ln [\omega / \alpha])^{2}}, & f=1 \\ B(f) \omega^{f-2}, & 1<f<2 \\ \text { constant }, & f=2 \\ C(f) \omega^{f-2}, & f>2\end{cases}
$$

with $\omega=\frac{f}{\lambda \rho}\left(E-\omega_{c}\right)$. This behavior is very uncommon, and seems to be particular to the choice of short-range single impurity potential, for long range one, one recovers the usual Lifshitz tail [4]. In the standard Lifshitz argument, when there is no magnetic field, low energy states are localized in regions of space where impurities are absent; An empty region, of typical size $\pi R^{2}$, contains states with energy of the order of $1 /\left(\pi R^{2}\right)$. For a Poisson distribution, the probability of not finding a single impurity in a volume $\pi R^{2}$ is $\exp \left(-\rho \pi R^{2}\right)$. Identifying the energy to the inverse size of the empty region let to obtain the low energy behavior $\exp \left(-\frac{\rho}{E}\right)$. This heuristic argument [5] has been later confirmed by an exact calculus [6, 7]. The question is whether it is possible to adapt this argument for the problem with strong magnetic field, in order to have a physical interpretation of the base of the spectrum, known from elsewhere to be constituated of localized states [8].

Let us consider the case where the density of impurities is less than the Landau degeneracy $(f<1)$. The zero energy delta peak has a simple interpretation [9] and corresponds to the delocalized state which is expected at the center of each Landau band [10, 11] :

these states are indeed linear combinations of Landau states, which vanish at the position of the impurities. And in a given volume $V$, the number of Landau states at disposal is $\rho_{l} V$. The number of constraints imposed on the zero energy states is $\rho V$, the number of impurities. As a consequence, the corresponding subspace of states has the dimension $\left(\rho_{l}-\rho\right) V$ (unless as will be seen later that two impurities coincide). This gives as expected the degeneracy $\rho_{l}(1-f)$ per unit volume, given by (11). What remains to be analysed is the $\omega^{-f}$ behavior of the excited states spectrum.

The paper is organized as follows, in the first part, the problem with a finite number $N$ of impurities is analysed in details. The zero modes are first extracted from the Hilbert space, which allows then to define the restriction of the Hamiltonian to the excited subspace as a $N \times N$ matrix. The two impurity case is explicitely solved and elucidates the mechanism which 
produces low energy states. The generalization to a cluster of impurities is then considered and an approximate expression of the lowest energy is found. In the second part, a statistical analyses is performed, using this expression, in order to find the most probable configurations corresponding to a given low energy, and the contribution to the DOS is computed in the case $f<1$.

\section{The $N$ delta impurity problem \\ a. Coherent states basis for the excited subspace}

The $N$ impurity problem, projected onto the LLL is defined by the Hamiltonian

$$
H=\lambda P_{0} \sum_{i=1}^{N} \delta\left(\mathbf{r}-\mathbf{r}_{i}\right) P_{0}
$$

after shifting the spectrum by a constant. $\lambda$ is the coupling constant of the delta potential, $P_{0}$ is the projection operator on the LLL. Let us consider the basis corresponding to the symmetric gauge, centered at position a (using complex notation and magnetic units) :

$$
\phi_{p}^{a}(\mathbf{r})=\frac{1}{\sqrt{\pi p !}}(z-a)^{p} e^{-\frac{1}{2}(z \bar{z}+a \bar{a}-2 z \bar{a})} \quad p \in N
$$

In the situation where there is only one impurity, situated at position $a$, these states remain

eigenstates, with zero energy for $p>0$ and with energy $\frac{\lambda}{\pi}$ for $p=0$. Let us associate to the impurity $i$ the coherent state $\psi_{i}$, corresponding to the only non-vanishing state at $\mathbf{r}_{i}$,

$$
\psi_{i}(\mathbf{r})=\phi_{0}^{z_{i}}(\mathbf{r})=\frac{1}{\sqrt{\pi}} e^{-\frac{1}{2}\left(z \bar{z}+z_{i} \bar{z}_{i}-2 z \bar{z}_{i}\right)}
$$

As already mentionned, the LLL is divided into two orthogonal subspaces : the zero energy subspace of dimension higher or equal to $\rho_{l} V-N$, and the excited subspace of dimension less or equal to $N$. The subspace of wave-functions vanishing at $\mathbf{r}_{i}$, is orthogonal to $\psi_{i}$ and contains the zero energy states. Therefore the zero-energy subspace is orthogonal to the one generated by $\psi_{1}, \ldots \psi_{N}$. Let us find under which conditions theses states are linearly independents.

Consider

$$
\psi(\mathbf{r})=\sum_{i=1}^{N} a_{i} \psi_{i}(\mathbf{r})
$$

a linear combination of these $N$ states. In the Landau symmetric basis $\psi$ has the form

$$
\psi(\mathbf{r})=\sum_{p=0}^{\infty} b_{p} \phi_{p}(\mathbf{r})
$$

The relation between the $b_{p}$ and $a_{i}$ is

$$
b_{p}=\frac{1}{\sqrt{p} !} \sum_{i=1}^{N} a_{i} \bar{z}_{i}^{p} e^{-\frac{1}{2} z_{i} \bar{z}_{i}}
$$

In order for $\psi$ to be identically zero, the $b_{p}$ have to vanish. In particular, imposing this to the first $N(p=0 \ldots N-1)$ leads to an homogeneous system of equations for the $a_{n}$, with a determinant proportionnal to the $\bar{z}_{i}$ 's Vandermonde determinant, i.e. a completely antisymmetric function of these variables. Therefore a necessary condition for the $\psi_{i}$ to be linearly dependent 
is that two impurities coincide, and it is evidently sufficient. As a consequence $\psi_{1}, \ldots \psi_{N}$ is a basis of the excited subspace (non-orthogonal).

Let us write the Hamiltonian into this basis. Starting from the decomposition (5) of an arbitrary excited state, the action of $H$ on this state is

$$
<\mathbf{r}|H| \psi>=\lambda \sum_{i=1}^{N} P_{0}\left(\mathbf{r}, \mathbf{r}_{i}\right) \sum_{n=1}^{N} a_{n} \psi_{n}\left(\mathbf{r}_{i}\right)
$$

with

$$
P_{0}\left(\mathbf{r}, \mathbf{r}^{\prime}\right)=\frac{1}{\pi} e^{-\frac{1}{2}\left(z \bar{z}+z^{\prime} \bar{z}^{\prime}-2 z \bar{z}^{\prime}\right)}
$$

the kernel of the LLL projection operator. Using the fact that

$$
<\psi_{i} \mid \psi_{j}>=\pi P_{0}\left(\mathbf{r}_{i}, \mathbf{r}_{j}\right)=\sqrt{\pi} \psi_{j}\left(\mathbf{r}_{i}\right)
$$

we obtain

$$
<\mathbf{r}|H| \psi>=\lambda \sum_{i=1}^{N} \sum_{j=1}^{N} a_{j} P_{0}\left(\mathbf{r}_{i}, \mathbf{r}_{j}\right) \psi_{i}(\mathbf{r})
$$

In conclusion the matrix elements of $H$ in the $\left(\psi_{1}, \ldots, \psi_{N}\right)$ basis are given by $\lambda P_{0}\left(\mathbf{r}_{i}, \mathbf{r}_{j}\right)$.

\section{b. Two impurities}

In the case with only two impurities, we can diagonalize this matrix. Choosing the spatial reference such that $z_{1}=-z_{2}=a / 2$, where $a$ is the distance between the two impurities, we have

$$
H_{2}=\frac{\lambda}{\pi}\left(\begin{array}{cc}
1 & e^{-\frac{1}{2} a^{2}} \\
e^{-\frac{1}{2} a^{2}} & 1
\end{array}\right)
$$

The eigenvalues of this matrix correspond to the energies $E_{-}$and $E_{+}$of the two excited states,

$$
E_{ \pm}=\frac{\lambda}{\pi}\left(1 \pm e^{-\frac{1}{2} a^{2}}\right)
$$

The corresponding wave-functions beeing (up to a normalization coefficient)

$$
\psi_{ \pm}=\psi_{1} \pm \psi_{2}=\frac{1}{\sqrt{\pi}} e^{-\frac{1}{2}\left(z \bar{z}+a^{2}\right)}\left(e^{2 a z} \pm e^{-2 a z}\right)
$$

In conclusion, when the two impurities are well seperated, the excited states have almost the same energy, comparable to the one impurity value. Whereas, a low energy state is obtained when the two impurities are close. For $a<<1$ this energy behave like

$$
E_{-} \simeq \frac{\lambda}{2 \pi} a^{2}
$$

\section{c. Impurity cluster}

The preceeding example suggests that low-energy states are associated with regions of high concentrations of impurities. Indeed, $N$ impurities involve $N$ localized states $\psi_{i}$ (which have a characteristic size $1 / \rho_{l}$ ). Low-lying states are expected to appear when the overlap between these states starts to be important. Consider a situation (figure 4 ) where $N$ impurities are 
located in a small volume $\left(\pi R^{2}\right)$, that is a cluster of impurities, then the $N$ corresponding states overlap essentially with the $N_{l}=\rho_{l} \pi R^{2}$ Landau states situated inside the disc (in the symmetric gauge, the states of the LLL are localized on a ring of radius $\sqrt{l / \rho_{l}} \pi$, where $l$ is the angular momentum [12]) So if $N>N_{l}$, we expect to have $N-N_{l}$ low-energy states. It seems therefore natural to consider such configurations in order to analyse the bottom of the spectrum.

Let us estimate the lowest energy corresponding to such a configuration. Consider the decompositions (5) and (6) of an excited state. A low energy state is supposed to avoid the impurities. A way to construct such a state, is to impose on the $b_{p}$ to vanish until $p=N-2$ included. In that case, $\psi$ has components only on the Landau states $p>N-2$, situated at a distance from the center of the cluster greater or equal to $\sqrt{\frac{N-1}{\pi \rho_{l}}}$. Such a state is given by the $a_{n}$, solution of the set of equations

$$
b_{p}=0=\sum_{n=1}^{N} a_{n} e^{-\frac{1}{2} z_{n} \bar{z}_{n}} z_{n}^{p} \quad p=0, \ldots N-2
$$

And the solution, up to a proportionality constant is

$$
a_{n} e^{-\frac{1}{2} z_{n} \bar{z}_{n}}=C_{N, n}
$$

where $C_{N, n}$ is the cofactor of the element $(N, n)$ in the Vandermonde type matrix:

$$
D_{N}^{p}=\left(\begin{array}{cccc}
1 & \ldots & \ldots & 1 \\
z_{1} & \ldots & \ldots & z_{N} \\
\vdots & \ldots & \ldots & \vdots \\
z_{1}^{N-2} & \ldots & \ldots & z_{N}^{N-2} \\
z_{1}^{p} & \ldots & \ldots & z_{N}^{p}
\end{array}\right)
$$

In particular, for $p=0 \ldots N-2$,

$$
\operatorname{det} D_{N}^{p}=0=\sum_{n=1}^{N} C_{N, n} z_{n}^{p}
$$

which is precisely what we want. Moreover the $C_{N, n}$ have the expression

$$
C_{N, n}=(-1)^{\frac{N(N-1)}{2}+n} \prod_{p<q p, q \neq n}\left(z_{p}-z_{q}\right)
$$

The matrix $H_{N}=\lambda P_{0}\left(\mathbf{r}_{i}, \mathbf{r}_{j}\right)$, written in $\psi_{1}, \ldots \psi_{N}$ basis, is self-adjoint and positive, so its smallest eigenvalue $E_{0}$ verify the inequality:

$$
E_{0} \leq \frac{\left(\psi \mid H_{N} \psi\right)}{(\psi \mid \psi)}
$$

with the norm defined by,

$$
(\psi \mid \psi)=\sum_{n=1}^{N} \bar{a}_{n} a_{n}
$$

From this choice and for the considered state the inequality rewrites

$$
E_{0} \leq E=\frac{\lambda}{\pi} \frac{\sum_{n, m} \bar{C}_{N, n} C_{N, m} e^{z_{m} \bar{z}_{n}}}{\sum_{n}\left|C_{N, n}\right|^{2} e^{\left|z_{n}\right|^{2}}}
$$


and

$$
E \leq \frac{\lambda}{\pi} \frac{\sum_{n, m} \bar{C}_{N, n} C_{N, m} e^{z_{m} \bar{z}_{n}}}{\sum_{n}\left|C_{N, n}\right|^{2}}
$$

Expanding the exponential in the preceeding expression, we observe that the first non-vanishing term corresponds to $\frac{\left(z_{m} \bar{z}_{n}\right)^{N-1}}{(N-1) !}$, because the determinant of $D_{N}^{p}$ is zero for $p<N-1$. In addition, since $\left|z_{n}\right|^{2} \leq N_{l} \leq N$, the serie has a rapid decay, which allow to neglect the remainder of the expansion. We then obtain

$$
E_{0} \leq \frac{\lambda}{\pi} \frac{1}{(N-1) !} \frac{\left|D_{N-1}\right|^{2}}{\sum_{n=1}^{N}\left|C_{N, n}\right|^{2}}
$$

with $D_{N-1}=(-1)^{\frac{N(N-1)}{2}} \prod_{p<q}\left(z_{p}-z_{q}\right)$ the Vandermonde determinant of the $z_{n}$ variables. If $n^{*}$ labels the impurity for which, $\prod_{p \neq n}\left|z_{n}-z_{p}\right|^{2}$ is minimum, then we have the inequality

$$
\sum_{n=1}^{N}\left|C_{N, n}\right|^{2} \geq N\left|C_{N, n^{*}}\right|^{2}
$$

which leads to the approximate form for $E_{0}$

$$
E_{0} \propto \frac{\lambda}{\pi} \frac{1}{N !} \min _{p} \prod_{n \neq p}\left|z_{p}-z_{n}\right|^{2}
$$

and which coincides with expression (15) in the two impurities case.

\section{Cluster thermodynamic}

We can now use this expression in order we understand the low energy behavior of the spectrum obtained by Brézin, Gross, Itzykson $(f<1)$. We start from the principle that each impurity in the system gives rise to an excited state with energy depending on the configuration of the other impurities. If the concentration around one impurity is high, in other words if the impurity is in a cluster, then the corresponding energy is low and not affected by the impurities situated outside of the cluster (too far away for overlap effect). We can therefore associate a low-energy state to the formation of a cluster around an impurity, and by extension, a density of states per impurity. Let $X_{i}$ be a variable parametrizing the cluster configuration of the impurity $i$. Its contribution to the density of state per impurity is proportionnal to the probability $P\left(X_{i}\right)$ of being realized

$$
\rho_{i}(E)=\int D X_{i} P\left(X_{i}\right) \delta\left(E\left(X_{i}\right)-E\right)
$$

So in average, the low-energy density of states by unit volume is proportional to $\rho$ times the preceeding expression. If we use now the expression (27) to evaluate the energy of the clusters, we see that to a given energy corresponds a statistical ensemble of clusters. Each cluster is defined by its volume $N_{l}$, its mean density $\nu=N / N_{l}>1>f$, and by the positions $z_{i}, i=1 \ldots N$, of the impurities in the cluster. At very low energy, the clusters are expected to be macroscopic objects, and have to be described by a finite number of macroscopic variables, giving the density profile, in replacement of the microscopic degrees of freedom (namely the individual positions of impurities). Let us look first for the distribution of positions in a cluster of energy $E$, size $N_{l}$ and mean density $\nu$. For a given configuration the energy is

$$
E=e^{\sum_{n=1}^{N} \log \left|z_{n}\right|^{2}-N \log N+N}
$$


using the Stirling formula $\left(N ! \simeq N^{N} e^{-N}\right)$ and with $0 \leq\left|z_{n}\right|^{2} \leq N_{l}\left(N_{l}=\pi \rho_{l} R^{2}\right)$. Consider a subdivision of the cluster in $M$ cells, corresponding to intervals of the $\left|z_{n}\right|^{2}$ equal to $a=N_{l} / M$ (cells with identical area $\frac{\pi R^{2}}{M}=\frac{a}{\rho_{l}}=\pi \delta r^{2}=\frac{\delta|z|^{2}}{\rho_{l}}$ ). If $n_{p}$ is the number of impurities in the cell $p$, then the probability associated to this configuration $\left(n_{1}, \ldots, n_{M}\right)$ of the cluster is

$$
P\left(n_{1}, \ldots, n_{M}, N\right)=\frac{N !}{n_{1} ! \ldots n_{M} !}\left(\frac{1}{M}\right)^{N} \frac{\left(f N_{l}\right)^{N}}{N !} e^{-f N_{l}}
$$

Since we are interested in the continuum limit, define $\left(x=|z|^{2} / N_{l}=\frac{p}{N_{l}} a\right)$

$$
\nu(x) d x=n_{p}=\nu(x) \frac{1}{M} \quad 1 \ll M \ll N
$$

the energy takes then the form

$$
\log E=N_{l} \int_{0}^{1}[\nu(x) \log x+\nu-\nu \log \nu] d x
$$

and, at leading contribution in $N_{l}$, the probability is

$$
\log P=N_{l} \int_{0}^{1}\left[\nu(x)\left(1-\log \frac{\nu(x)}{f}\right)-f\right] d x
$$

with the constraint

$$
\int_{0}^{1} \nu(x) d x=\nu
$$

Let us determine the configuration for which $\log P$ is maximum at fixed $E, N_{l}$ and $\nu$. Using a Lagrange multiplier for the energy constraint we obtain the saddle point equation

$$
\frac{\partial \log P}{\partial \nu(x)}-\alpha \frac{\partial \log E}{\partial \nu(x)}=0
$$

The solution, with proper normalization, is

$$
\nu(x)=\nu(1-\alpha)\left(\frac{x}{N_{l}}\right)^{-\alpha}
$$

$\alpha$ being implicitely determined through the relation between $\gamma=\frac{1}{1-\alpha}$ and the energy,

$$
\log E=-N_{l}(\nu(\log \nu-1)+\gamma \nu)
$$

and the probability now takes the form

$$
\log P=\log E-N_{l}(f-\nu(1+\log \gamma f))
$$

At a given energy, the possible configurations are parametrized by $\left(N_{l}, \nu\right)$. The saddle point is determined by the set of equations

$$
\begin{aligned}
& \left(\frac{\partial \log P}{\partial N_{l}}\right)_{\nu, E}=0 \\
& \left(\frac{\partial \log P}{\partial \nu}\right)_{N_{l}, E}=0
\end{aligned}
$$


Using (37), which determines $\gamma$ these equations rewrites

$$
\begin{gathered}
\log \gamma f+\frac{1-\log \nu}{\gamma}-\frac{f}{\nu}=0 \\
\log \gamma f-\frac{1}{\gamma} \log \nu=0
\end{gathered}
$$

So finally, $\log P$ has its maximum (which can be verified by computing second derivatives) at energy $E$ when $\nu=1, N_{l}=-f \log E /(1-f)$, which corresponds to $\gamma=1 / f$. The other solution ( $\nu=f$ and $\gamma=1$ is also a local maximum, but outside the range of interest for the parameters. For this type of configurations (paramatrized now only by $X=N_{l}$ ), we have the relation

$$
\log \frac{P}{E}=-f \log E
$$

Using (28) (with the change of variable $D X \propto d E / E$ ), we arrive at the expected low-energy behavior of the density of states

$$
\rho(E) \propto E^{-f}
$$

Moreover, states contributing to this behavior are associated to the existence of impurity clusters of size $N_{l}=-f \log E /(1-f)$ and the shape

$$
\nu(x)=f x^{f-1}
$$

In contrary to the Lifshitz argument, the low energy states are associated with regions of high impurity concentration around which they localize, with caracteristic size $\log \frac{1}{E}$, a rough indication of a logarithmic divergency of the localization length, at least when $f \ll 1$. This feature might be very particular to the zero-range nature of the impurity scattering potential. When $f$ approaches 1, this picture might be modified by some "percolation" effect of these cluster. For $f$ greater than 1 the argument developped in this paper is not applicable, neither is the stantard Lifshits argument, to reproduce the low energy spectrum. This seems to indicate that states are not localized at the bottom of the spectrum in this case.

\section{Acknowledgements}

I am gratful to A. Comtet for interesting me to this question and for useful discussions, as well as to J.M. Leinaas, L. Pastur and S. Villain-Guillot. I am thankful to the Institute of physics of the Oslo University for hospitality, and the National Reasearch Council of Norway for financial support.

\section{References}

[1] F. Wegner, Z Physik. B 51 (1984) 279

[2] E. Brezin, D. Gross, C. Itzykson, Nucl. Phys. B 235 (1984) 24

[3] A. Klein, J.F. Perez, Nucl. Phys. B 251 (1985) 199

[4] K. Broderix, D. Hundertmark, W. Kirsch, H. Leschke, J Stat. Phys. 80 (1995) 1

[5] I.M. Lifshitz, Usp. Fiz. Nauk. 83 (1964) 617 
[6] J. M. Luttinger, R. Tao, Ann. Phys. 145 (1983) 185

[7] J. M. Luttinger, J. Waxler, Ann. Phys. 175 (1987) 319

[8] T.C. Dorlas, N. Macris, S.V. Pulé, J Stat. Phys. 87 (1997) 847

[9] M. Ya. Azbel', Physica A 200491

[10] A.M.M. Pruisken, Phys. Rev. Lett. 61 (1988) 1297

[11] Y. Avishai, R.M. Redheffer, Y.B. Band, J Phys. A 235 (1992) 3883

[12] C. Itzykson, J.M. Drouffe, Statistical Field Theory, Cambridge University Press (1989) 